\title{
Simultaneous estimation of astrophysical and cosmological stochastic gravitational-wave backgrounds with terrestrial detectors
}

\author{
Katarina Martinovic $\odot,{ }^{1, *}$ Patrick M. Meyers $\odot,{ }^{2,3, \dagger}$ Mairi Sakellariadou $\odot,{ }^{1,4, \$}$ and Nelson Christensen $\circledast^{5, \S}$ \\ ${ }^{1}$ Theoretical Particle Physics and Cosmology Group, Physics Department, King's College London, \\ University of London, Strand, London WC2R 2LS, United Kingdom \\ ${ }^{2}$ School of Physics, University of Melbourne, Parkville, VIC 3010, Australia \\ ${ }^{3}$ OzGrav, University of Melbourne, Parkville, VIC 3010, Australia \\ ${ }^{4}$ CERN, Theoretical Physics Department, Geneva 23, 1211 Switzerland \\ ${ }^{5}$ Artemis, Université Côte d'Azur, Observatoire de la Côte d'Azur, CNRS, Nice 06300, France
}

(Received 11 November 2020; accepted 12 February 2021; published 26 February 2021)

\begin{abstract}
The recent Advanced LIGO and Advanced Virgo joint observing runs have not claimed a stochastic gravitational-wave background detection, but one expects this to change as the sensitivity of the detectors improves. The challenge of claiming a true detection will be immediately succeeded by the difficulty of relating the signal to the sources that contribute to it. In this paper, we consider backgrounds that comprise compact binary coalescences and additional cosmological sources, and we set simultaneous upper limits on these backgrounds. We find that the Advanced LIGO/Advanced Virgo network, operating at design sensitivity, will not allow for separation of the sources we consider. Third-generation detectors, sensitive to most individual compact binary mergers, can reduce the astrophysical signal via subtraction of individual sources, and potentially reveal a cosmological background. Our Bayesian analysis shows that, assuming a detector network containing Cosmic Explorer and Einstein Telescope and reasonable levels of individual source subtraction, we can detect cosmological signals $\Omega_{\mathrm{CS}}(25 \mathrm{~Hz})=4.5 \times 10^{-13}$ for cosmic strings, and $\Omega_{\mathrm{BPL}}(25 \mathrm{~Hz})=2.2 \times 10^{-13}$ for a broken power-law model of an early Universe phase transition.
\end{abstract}

DOI: 10.1103/PhysRevD.103.043023

\section{INTRODUCTION}

A stochastic gravitational-wave background (SGWB) is a random signal produced by many weak, independent and unresolved sources; it can be of a cosmological or astrophysical origin. A variety of early Universe processes, like quantum vacuum fluctuations during inflation, postinflationary preheating, first-order phase transitions, or topological defects (in particular cosmic strings) can lead to a SGWB [1-3].

An astrophysical contribution to the SGWB comes from the superposition of unresolved gravitational-wave (GW) sources of stellar origin. This includes burst sources, like core-collapse supernovae and the final stage of compact binary coalescences $(\mathrm{CBCs})$, together with quasiperiodic long-lived sources like pulsars and the early inspiral phase

\footnotetext{
*katarina.martinovic@kcl.ac.uk pat.meyers@unimelb.edu.au *mairi.sakellariadou@kcl.ac.uk §nelson.christensen@oca.eu
}

Published by the American Physical Society under the terms of the Creative Commons Attribution 4.0 International license. Further distribution of this work must maintain attribution to the author(s) and the published article's title, journal citation, and DOI. of compact binaries [4,5]. A detection of a SGWB can provide important astrophysical information about, for instance, the mass range for neutron star and black hole progenitors, or the rate of compact binaries [6]. It also sheds light on particle physics models beyond the Standard Model and the early stages of our Universe. Advanced LIGO's second observing run saw no SGWB, and placed upper limits on frequency-independent and $\mathrm{CBC}$ backgrounds, as well as additional GW polarizations [7].

Once a SGWB is successfully detected, there will be the challenge of identifying the sources that contribute to it. Untangling these signals will deepen our knowledge of merger rates and population models [8-11], our understanding of exotic objects [12-15] and in particular early Universe models [3,16-18]. Combining a stochastic analysis with information from individual events may provide the appropriate means to learn about GW sources [19].

We have recently developed a parameter estimation analysis to distinguish between correlated magnetic noise that contaminates terrestrial GW detectors and a real GW signal [20]. The low-frequency resonances in the Earth's global electromagnetic field could couple to the mass suspension system and electronics in the detectors, and mimic a SGWB. The method presented in Ref. [20] helps to minimize the possibility of a false detection. In this paper, 
we adapt that method, which is based on techniques already present in the literature [21-23], to separate the astrophysical and cosmological contributions to the SGWB. We first discuss second-generation detectors like Advanced LIGO [24] and Advanced Virgo [25]. We then move to thirdgeneration GW detectors, namely Einstein Telescope [26] and Cosmic Explorer [27,28], and comment on how the study could be adapted to the space-based LISA detector. The future detector networks require subtraction of the known compact binaries background from the stochastic signal, prior to the parameter estimation [29-33], to ensure that the cosmological background is not obscured by the astrophysical one.

In Sec. II we discuss the individual sources we choose for this study. We describe the GW signals injected and the analysis we perform in Sec. III. We summarize our results in Sec. IV and make concluding remarks in Sec. V.

\section{SGWB SOURCES}

In this section, we discuss three different potential contributions to a SGWB. We discuss the astrophysical contribution from $\mathrm{CBCs}$, and two cosmological sources of GWs. We consider cosmic strings, which are onedimensional topological defects [34], and an earlyUniverse first-order phase transition $[35,36]$.

\section{A. CBC background}

The CBC background is likely to be the largest contribution to the SGWB [6]. Therefore, any attempt to measure other contributions to the background should be done in such a way as to simultaneously measure a CBC background and other contributions. The analytic model describing the $\mathrm{CBC}$ background depends on quantities such as redshift and merger rates [4,6]; the inspiral phase can be approximated as

$$
\Omega_{\mathrm{CBC}}(f)=\Omega_{2 / 3}\left(\frac{f}{25 \mathrm{~Hz}}\right)^{2 / 3} .
$$

In the case of second-generation detectors, we can use this approximation freely $[37,38]$. When it comes to future GW detectors, however, the approximation cannot be applied to the entire frequency band. Instead, one must also include the contributions from the merger and ringdown phases that cause measurable deviations from this approximation $[30,38]$. For the purpose of this study, we restrict ourselves to the range $(10-100) \mathrm{Hz}$, the frequency range over which the approximation in Eq. (1) is valid, even after individual source subtraction [30].

The current estimate of the amplitude of the CBC spectrum from individual sources over the Advanced LIGO and Advanced Virgo frequency range is $\Omega_{\mathrm{CBC}}=$ $1.8_{-1.7}^{+2.7} \times 10^{-9}$, with $90 \%$ confidence, at a reference frequency of $25 \mathrm{~Hz}$ [6]. This estimate includes contributions from binary black holes, binary neutron stars, and black hole-neutron star systems.

There are numerous studies on subtracting resolvable CBC signals from the data, and these can lead to a reduction in their contribution to the SGWB by as much as 2 orders of magnitude for binary black hole signals and 1 order of magnitude for binary neutron star signals [29-33]. When considering future detectors like Einstein Telescope [26] and Cosmic Explorer [27,28], we assume a scenario where such a subtraction has already been made, following the results from Ref. [30].

\section{B. Cosmic strings}

A phase transition followed by a spontaneously broken symmetry can leave behind topological defects as remnants of a previous more symmetric phase. One particular class of such defects is cosmic strings (CSs), line-like defects, generically formed within the context of grand unified theories [39].

A network of cosmic strings is mainly characterized by the string tension $G \mu$, where $G$ is Newton's constant, and $\mu$ is the mass per unit length. The dynamics of a string network are driven by the formation of loops and the emission of bursts of GWs, predominantly from cusps and kinks. The superposition of these bursts leads to a SGWB over a large range of frequencies, making it a target for $\mathrm{GW}$ searches from pulsar timing arrays in the $\mathrm{nHz}$ band as well as the ground-based detectors we consider here [40-42].

In the high-frequency regime we consider, $(10-100) \mathrm{Hz}$, the spectrum of the SGWB is flat, i.e., $\Omega_{\mathrm{CS}}(f)=$ const [43], and it only depends on the averaged total power emitted by a loop, and the total number of loops. A SGWB analysis can thus put a limit on the string tension, and consequently on the energy scale of the phase transition leading to the formation of these objects.

The 95\% credible upper limit placed after the first two LIGO observation runs, assuming a uniform prior, is $\Omega_{\mathrm{CS}}=6.0 \times 10^{-8}$ [7]. This implies upper bounds to the string tension, $G \mu \leq 1.1 \times 10^{-6}$ and $G \mu \leq 2.1 \times 10^{-14}$, for the loop distribution models in Refs. [44] and [45], respectively.

\section{First-order phase transitions}

If a phase transition occurred at temperatures $\left(10^{4}-10^{5}\right) \mathrm{TeV}$, the corresponding GW spectrum would be observed in the (10-100) $\mathrm{Hz}$ frequency range we consider [46]. The phase transition associated with the breaking of Peccei-Quinn symmetry, for instance, could have happened at such high temperatures, leading to the QCD axion, a well-motivated extension to the Standard Model. In this scenario, the growth of the true vacuum bubbles, and their subsequent collisions, give out GWs due to several effects [47]. The strongest of those is most likely due to sound waves from bubble growth in plasma. 
The turbulence of the plasma in which the bubbles grow can also produce GWs. Finally, GWs are emitted due to collision of the scalar wall profiles. There exist numerical [48-50] and analytic [51,52] models for the shape of $\Omega_{\mathrm{GW}}$ as a function of frequency for each of these contributions.

The frequency spectrum of the SGWB produced by most models can be captured by a smoothed broken power law (BPL):

$$
\Omega_{\mathrm{BPL}}=\Omega_{*}\left(\frac{f}{f_{*}}\right)^{\alpha_{1}}\left[1+\left(\frac{f}{f_{*}}\right)^{\Delta}\right]^{\left(\alpha_{2}-\alpha_{1}\right) / \Delta} .
$$

For example, numerical simulations find the GW spectrum due to the sound waves in the plasma [53]

$$
h^{2} \Omega_{\mathrm{SW}}(f)=F\left(\beta, H_{*}, \kappa_{\mathrm{sw}}, \alpha, g_{*}, v_{w}\right) \frac{\left(f / f_{\mathrm{sw}}\right)^{3}}{\left[1+0.75\left(f / f_{\mathrm{sw}}\right)^{2}\right]^{7 / 2}},
$$

where $\beta$ is the transition strength, $H_{*}$ is the Hubble constant at the time of GW production, $\kappa_{\mathrm{sw}}$ is the efficiency factor, $\alpha$ is the ratio of latent heat released in the phase transition to the heat of the radiation bath, $g_{*}$ is the number of relativistic degrees of freedom, $v_{w}$ is the bubble wall velocity, and $f_{\mathrm{sw}}=f_{\mathrm{sw}}\left(\beta, H_{*}\right)$ is the peak frequency.

If we use Eq. (2) to approximate Eq. (3), then we have $\alpha_{1}=3, \alpha_{2}=-4$ and $\Delta=2$. Relating $\Omega_{*}$ and $f_{*}$ to the long list of physical parameters that control the phase transition is beyond the scope of this study.

\section{MODEL SELECTION AND PARAMETER ESTIMATION}

We undertake a Bayesian parameter estimation and model selection study. For a single GW detector pair, $i j$, the log-likelihood is

$$
\begin{aligned}
\log p\left(\hat{C}_{i j}(f) \mid \boldsymbol{\theta}_{\mathrm{GW}}\right)= & -\frac{1}{2} \sum_{f} \frac{\left[\hat{C}_{i j}(f)-\Omega_{\mathrm{GW}}\left(f, \boldsymbol{\theta}_{\mathrm{GW}}\right)\right]^{2}}{\sigma_{i j}^{2}(f)} \\
& -\frac{1}{2} \sum_{f} \log \left[2 \pi \sigma_{i j}^{2}(f)\right]
\end{aligned}
$$

where $\Omega_{\mathrm{G} W}(f)$ is the model spectrum and $\boldsymbol{\theta}_{\mathrm{GW}}$ are the parameters that define the model. The cross-correlation estimator, $\hat{C}_{i j}(f)$, is calculated from detector data and was discussed in detail in Refs. [7,20,54]. We extend this analysis to include three GW detectors by adding log likelihoods for the individual pairs to construct a multiplebaseline log likelihood.

To compare two models, $\mathcal{M}_{1}$ and $\mathcal{M}_{2}$, and make statements about which is more favored by the data, we utilize Bayes factors,

$$
\mathcal{B}_{\mathcal{M}_{2}}^{\mathcal{M}_{1}}=\frac{\int \mathrm{d} \boldsymbol{\theta} p\left(\hat{C}_{i j}(f) \mid \boldsymbol{\theta}, \mathcal{M}_{1}\right) p\left(\boldsymbol{\theta} \mid \mathcal{M}_{1}\right)}{\int \mathrm{d} \boldsymbol{\theta} p\left(\hat{C}_{i j}(f) \mid \boldsymbol{\theta}, \mathcal{M}_{2}\right) p\left(\boldsymbol{\theta} \mid \mathcal{M}_{2}\right)}
$$

where $p(\boldsymbol{\theta} \mid \cdot)$ is the prior probability of our parameters given a choice of model. The integrand in Eq. (5) is the joint posterior distribution of the model parameters, which is evaluated as part of the evaluation of the Bayes factors.

For large and positive values of $\ln \mathcal{B}_{\mathcal{M}_{2}}^{\mathcal{M}_{1}}$, there is strong evidence for $\mathcal{M}_{1}$ over $\mathcal{M}_{2}$. Likewise, large and negative values show preference for $\mathcal{M}_{2}$. Relating this quantity to a frequentist signal-to-noise ratio (SNR) statistic [1], we have $\ln \mathcal{B} \propto \mathrm{SNR}^{2}$ [54]. We use the nested sampler DYNESTY through the front-end package BILBY to evaluate Bayes factors for our models, as well as posterior distributions on the parameters.

While the posterior distribution of $\boldsymbol{\theta}_{\mathrm{GW}}$ is evaluated in conjunction with Bayes factors, we can also analytically calculate a bound on covariance between model parameters using the information matrix. This has been used for estimating parameter covariance for SGWB models in other studies as well $[38,55,56]$. For the case of a Gaussian likelihood with uncorrelated measurements (frequency bins) with an unbiased estimator, the information matrix is given by

$\mathcal{I}_{i j}(\theta)=\sum_{f} \sigma(f)^{-2}\left(\frac{\partial \Omega_{\mathrm{GW}}(f, \theta)}{\partial \theta_{i}}\right)\left(\frac{\partial \Omega_{\mathrm{GW}}(f, \theta)}{\partial \theta_{j}}\right)$.

The covariance between model parameters is theoretically bounded below by the inverse of the information matrix

$$
\operatorname{cov}_{\theta}\left(\theta_{i}, \theta_{j}\right) \geq\left[\mathcal{I}^{-1}(\theta)\right]_{i j}
$$

This bound, known as the Cramér-Rao lower bound, can be exceeded by including, e.g., informative prior information. However, the structure of the information matrix can still offer valuable insight into the degeneracy of certain model parameters with one another and offer an intuitive picture of the parameter estimation problem.

\section{A. Injected signal}

We consider two types of injections: one containing a $\mathrm{CBC}$ and a cosmic string, and another one containing a $\mathrm{CBC}$ and a background due to phase transitions; see Table I. The background labeled here as $\mathrm{CBC}$ refers to what is left once we subtract the known CBC contribution,

TABLE I. GW spectra injected, and the parameters estimated in the analysis.

\begin{tabular}{lcc}
\hline \hline & $\Omega_{\mathrm{GW}}(f)$ & GW parameters, $\boldsymbol{\theta}_{\mathrm{GW}}$ \\
\hline Injection 1 & $\Omega_{\mathrm{CBC}}(f)+\Omega_{\mathrm{CS}}(f)$ & $\left(\Omega_{2 / 3}, \Omega_{\mathrm{CS}}\right)$ \\
Injection 2 & $\Omega_{\mathrm{CBC}}(f)+\Omega_{\mathrm{BPL}}(f)$ & $\left(\Omega_{2 / 3}, \Omega_{*}, f_{*}\right)$ \\
\hline \hline
\end{tabular}


i.e., it is the unresolved astrophysical background. For the second injection, we choose a broken power law with exponents $\alpha_{1}=3, \alpha_{2}=-4$, and $\Delta=2$ which best describes $\Omega_{\mathrm{SW}}$, the sound-wave contribution to $\Omega_{\mathrm{GW}}$. In this case our Bayesian search estimates the peak frequency, $f_{*}$, as well as the amplitude of the smooth broken power law, $\Omega_{*}$.

The injection strengths we choose vary from one detector network to another. The instrumental noise is included at the level of the design sensitivity curves of the detectors. We consider O4 sensitivity for Advanced LIGO and Advanced Virgo [57], ET-D for the Einstein Telescope [58] and CE Wideband for the Cosmic Explorer [59]. The same prior is used for the recovered amplitudes, $\Omega_{2 / 3}, \Omega_{\mathrm{CS}}, \Omega_{*}$, all of them $\log$ uniformly distributed between $10^{-15}$ and $10^{-8}$. All results are presented for 1 year observation time.

\section{RESULTS}

We present results on source separation for a SGWB detection with different sets of GW detector networks.

\section{A. Advanced LIGO and Advanced Virgo}

In this section we consider the separation of a CS signal from a CBC signal with the current detector network operating at design sensitivity. We vary injection strengths, with 25 injections log-uniformly distributed between $\Omega_{2 / 3} \in\left(10^{-9.4}, 10^{-8.4}\right)$. These values were chosen by using $90 \%$ limits on CBC background from Ref. [6]. We explore the flat cosmic string spectrum with 35 injections loguniformly distributed between $\Omega_{\mathrm{CS}} \in\left(10^{-9.4}, 10^{-7.4}\right)$. The upper limit of the injection range is consistent with constraints placed on a cosmic string SGWB spectrum from data in the first two observational runs [7]. The Bayes factors we find are too low to differentiate between the signals, with values ranging between -0.1 and 0.1 , indicating that one cannot distinguish models that include both spectra from models that include only a CBC background. Other methods, which seek to model the contribution from individual CBCs on shorter time scales, along with an isotropic, flat background propose ways of overcoming these obstacles $[60,61]$.

\section{B. Third-generation detectors}

Operating at their design sensitivity, the Advanced LIGO/Advanced Virgo network cannot achieve source separation of a detected stochastic signal. We therefore pursue studies in future detectors. As was done in Ref. [30], we consider a network of Cosmic Explorer detectors at the Hanford and Livingston locations, and Einstein Telescope at the Virgo site. Figure 1 in Ref. [30] estimates that after individual source subtraction, the residual CBC contribution to the SGWB is dominated by unresolved binary neutron star mergers at the level of $\sim 10^{-11}$ at $10 \mathrm{~Hz}$. We therefore use a log-uniformly distributed range of
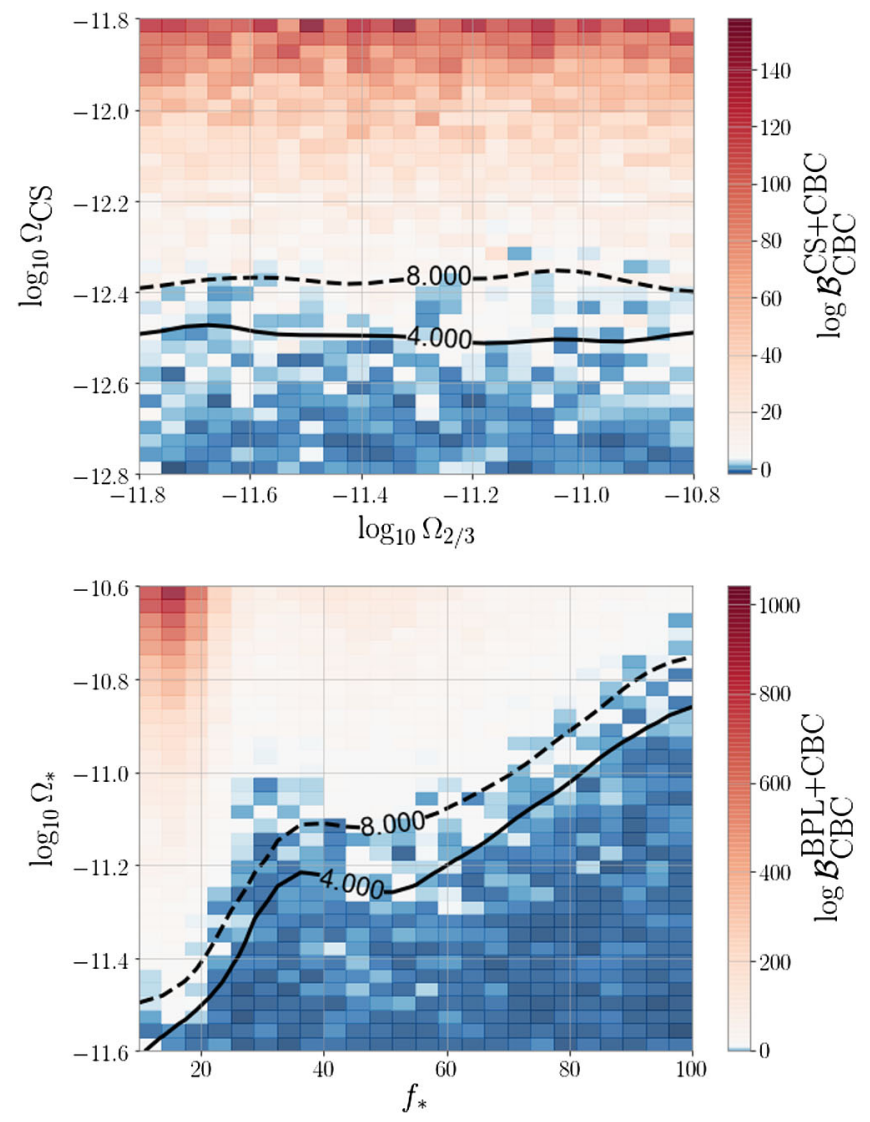

FIG. 1. Variation of $\log$ Bayes factor with the injected power laws for cosmic strings (top panel) and a first-order phase transition (bottom panel) for third-generation detectors. The residual CBC amplitude for the bottom panel is $\Omega_{2 / 3}=1 \times$ $10^{-11}$ for all of the injections. The contour plots show values of $\log \mathcal{B}=4$ or $\log \mathcal{B}=8$, which is roughly when we start to see significant preference for one of the models, since $\log \mathcal{B}=8$ corresponds to approximately $\mathrm{SNR}=4$.

$\Omega_{2 / 3} \in\left(10^{-11.8}, 10^{-10.8}\right)$ at $25 \mathrm{~Hz}$ in the top panel of Fig. 1, and fix $\Omega_{2 / 3}=1 \times 10^{-11}$ for all the injections in the bottom panel. We then use comparable signal strengths for the cosmological contributions, in particular $\Omega_{\mathrm{CS}} \in$ $\left(10^{-12.8}, 10^{-11.8}\right)$ and $\Omega_{*} \in\left(10^{-11.6}, 10^{-10.6}\right)$.

The GW selection effect could favor the detection of the best oriented and located sources, especially at larger redshift, disqualifying the assumption of an isotropic SGWB in the standard cross-correlation statistic. This leads to a systematic bias in the residual background and hence to a correction for the overlap reduction function $[62,63]$. This could provide another way of discriminating between an astrophysical and a cosmological background which we will investigate in a future study.

From the top panel of Fig. 1, we see that we start to confidently separate a flat spectrum from the residual CBC signal for $\Omega_{\mathrm{CS}}=4.5 \times 10^{-13}$. Cosmic string backgrounds lower than this get lost in the unrecovered CBCs and cannot be singled out. Our sensitivity allows constraints to be 
placed on the string tension as low as $G \mu \leq 3.0 \times 10^{-17}$ and $G \mu \leq 4.0 \times 10^{-19}$, for the cosmic string loop distribution models in Refs. [44] and [45], respectively. Similar sensitivity to a cosmic string spectrum is expected from the space-based LISA detector, whereas the Square Kilometer Array is expected to at most probe $G \mu$ values 3 or 4 orders of magnitude less sensitive [64].

As for a broken power law background due to an early Universe phase transition, we find that the Cosmic Explorer and Einstein Telescope network's sensitivity is highly dependent on the break frequency of the spectrum; see bottom panel of Fig. 1. The most conservative estimate we find of a detectable BPL signal (i.e., with $\log \mathcal{B}=8$ ), is the one associated with $f_{*}=100 \mathrm{~Hz}, \Omega_{*}=1.8 \times 10^{-11}$.
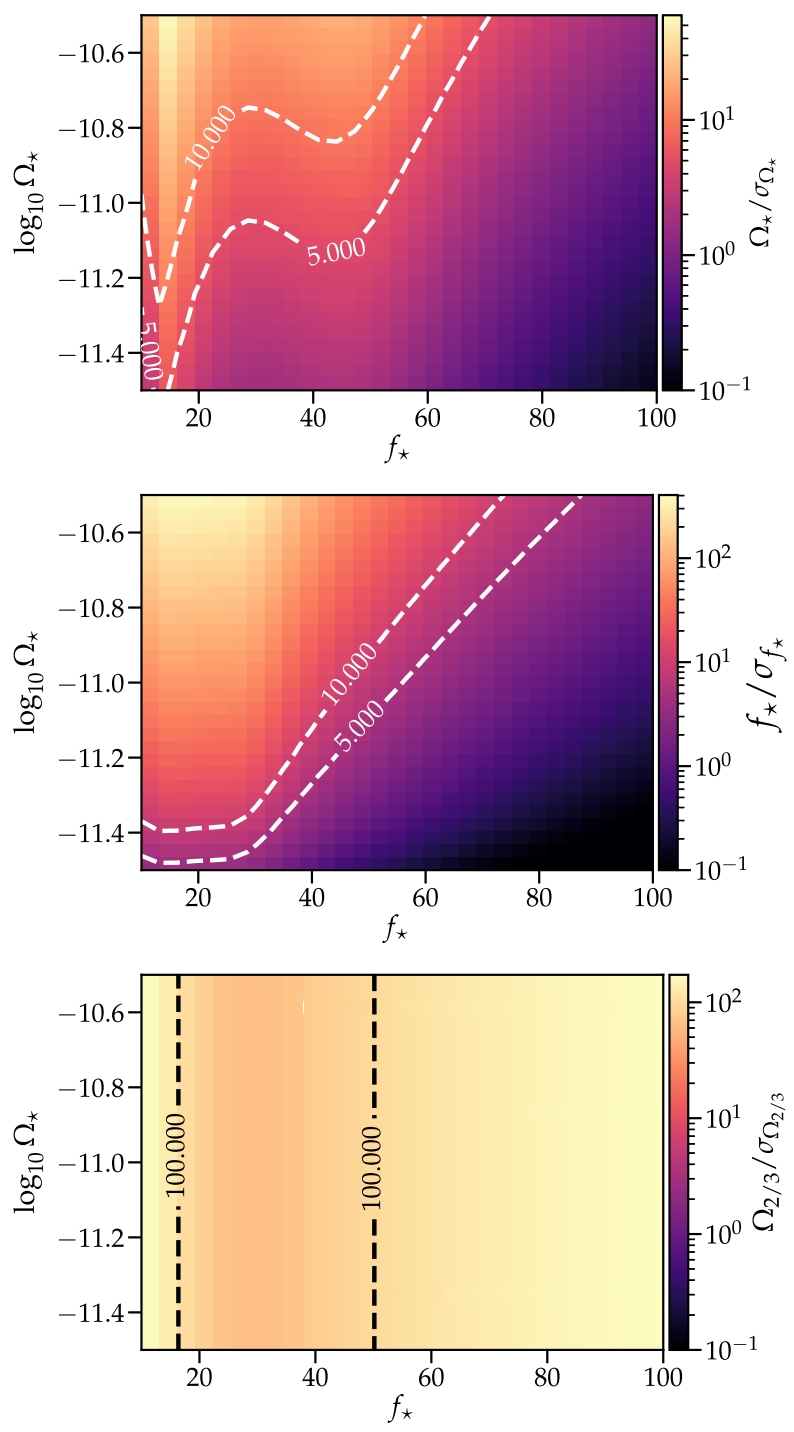

FIG. 2. Precision with which we can measure $f_{*}, \Omega_{*}$, and $\Omega_{2 / 3}$ for the broken power law model, where $\sigma$ for each parameter is estimated using the bound in Eq. (7). The model parameter used for $f_{*}$ and $\Omega_{*}$ is given by the value of the $x$ and $y$ axes respectively. The residual $\mathrm{CBC}$ injection is $\Omega_{2 / 3}=1 \times 10^{-11}$ for all simulations.
Taking into account injected values for $\alpha_{1}, \alpha_{2}, \Delta, f_{*}$, we estimate a stochastic signal of amplitude $\Omega_{\mathrm{BPL}}=2.2 \times$ $10^{-13}$ at $25 \mathrm{~Hz}$.

We also look at the precision with which we can measure $\Omega_{*}, f_{*}$ and $\Omega_{2 / 3}$ using the covariance bound in Eq. (7). We use $f_{*} / \sigma_{f_{*}}$ as a proxy for the precision of our $f_{*}$ measurement, with $\sigma_{f_{*}}=\left[\operatorname{cov}\left(f_{*}, f_{*}\right)\right]^{1 / 2}$ estimated from Eq. (7) (and analogous expressions for $\Omega_{*}$ and $\Omega_{2 / 3}$ ). In Fig. 2, we show the theoretical bound on this precision for $\Omega_{*}, f_{*}, \Omega_{2 / 3}$ as a function of the strength and shape of the broken power law background. In all three panels, the horizontal axis is $f_{*}$ and the vertical axis is $\log _{10} \Omega_{*}$. The color is the precision statistic discussed above. For all three panels, we have fixed $\Omega_{2 / 3}=1 \times 10^{-11}$. The broken power law model parameters are best estimated when $f_{*} \approx 20 \mathrm{~Hz}$ and are improved as $\Omega_{*}$ increases. Interestingly, the theoretical precision with which we measure $\Omega_{2 / 3}$ is independent of $\Omega_{*}$, but is dependent upon the shape, which is governed by $f_{*}$. This is because elements of the information matrix in Eq. (6) that involve derivatives of $\Omega_{*}$ and $\Omega_{2 / 3}$ are independent of both $\Omega_{*}$ and $\Omega_{2 / 3}$ because these variables appear linearly in separate terms of the combined model $\Omega_{\mathrm{BPL}}(f)+\Omega_{\mathrm{CBC}}(f)$. This means that the variances of $\Omega_{*}$ and $\Omega_{2 / 3}$, and the covariance between them are independent of these amplitudes. These variables are still correlated with each other, but the covariance between them is independent of the values themselves.

\section{DISCUSSION}

We have looked at current, and future, terrestrial GW detectors to see if we can successfully perform source separation of a detected SGWB signal. This is an important task, since it allows us to relate a detection to physical theories underlying it and perhaps give us a hint of beyond-Standard-Model physics. Although Advanced LIGO and Advanced Virgo sensitivity is not sufficient to separate sources, we found promising results for the thirdgeneration of detectors such as the Einstein Telescope [26] and Cosmic Explorer [27,28].

Our study concerned the frequency range for groundbased detectors. However, our methods will certainly be applicable for the future space-based detector, LISA [65]. The LISA observational band offers an exciting possibility to observe GWs from phase transitions [47]. Much work has been done to develop methods to characterize an arbitrary SGWB spectrum [66], as well as techniques to distinguish a cosmologically produced SGWB from galactic binaries [67], a binary black hole produced background [60], and instrumental noise $[67,68]$. A similar spectral separation study for LISA would be more complicated due to the nature of the time delay interferometry [69] and the necessity to simultaneously estimate the LISA noise. As such, we will apply the methods we have developed to LISA in a future study. 
This analysis can be additionally extended by considering other cosmological sources of a SGWB. One can for instance consider the minimal pre-big-bang model for which $\Omega_{\mathrm{GW}}(f)$ today scales as $f^{3}$ at the low-frequency end of the spectrum, whereas in the high-frequency range its behavior depends on a dimensionless free parameter of the model [70]. Furthermore, one can consider the full analytical model for a $\mathrm{CBC}$ background, thereby expanding the studied frequency range.

Let us note that we have not considered Schumann noise, which could contaminate the stochastic background leading to a false detection [71]. This issue may be of concern for LIGO/Virgo, but as discussed above, there can be no source separation for current detectors. Einstein Telescope is expected to have weaker coupling to Schumann noise due to heavier test masses, and the predicted magnetic budget is well below the sensitivity curves for post-Wiener filtering [72]. Investigation of the magnetic budget for the Cosmic Explorer has not been undertaken, and there is uncertainty over what the magnetic contamination will look like. As studies on third-generation detectors advance, we plan to extend our work with a detailed treatment of correlated magnetic noise.

\section{ACKNOWLEDGMENTS}

The authors would like to thank the LIGO/Virgo Stochastic Background group for helpful comments and discussions, in particular Alexander Jenkins for his numerical simulations of cosmic string spectra, and Tania Regimbau for useful discussions. The authors are grateful for computational resources provided by the LIGO Laboratory and supported by National Science Foundation Grants No. PHY-0757058 and PHY0823459. Parts of this research were conducted by the Australian Research Council Centre of Excellence for Gravitational Wave Discovery (OzGrav), through Project No. CE170100004. K. M. is supported by King's College London through a Postgraduate International Scholarship. M. S. is supported in part by the Science and Technology Facility Council (STFC), United Kingdom, under the research Grant No. ST/P000258/1. N. C. acknowledges support from National Science Foundation Grant No. PHY1806990. This paper has been given LIGO DCC number P2000470. Numerous software packages were used in this paper. These include Matplotlib [73], Numpy [74], scipy [75], BILBY [76], cpnest [77], ChainConsumer [78], and seaborn [79].
[1] B. Allen, The stochastic gravity-wave background: Sources and detection, in Relativistic Gravitation and Gravitational Radiation. Proceedings, School of Physics, Les Houches, France, 1995, edited by J.-A. Marck and J.-P. Lasota, Cambridge Contemporary Astrophysics (Cambridge University Press, Cambridge, England, 1997), pp. 373-417.

[2] M. Maggiore, Stochastic backgrounds of gravitational waves, ICTP Lect. Notes Ser. 3, 397 (2001).

[3] C. Caprini and D. G. Figueroa, Cosmological backgrounds of gravitational waves, Classical Quantum Gravity 35, 163001 (2018).

[4] T. Regimbau, The astrophysical gravitational wave stochastic background, Res. Astron. Astrophys. 11, 369 (2011).

[5] N. Christensen, Stochastic gravitational wave backgrounds, Rep. Prog. Phys. 82, 016903 (2019).

[6] B. P. Abbott et al., GW170817: Implications for the Stochastic Gravitational-Wave Background from Compact Binary Coalescences, Phys. Rev. Lett. 120, 091101 (2018).

[7] B. P. Abbott et al., Search for the isotropic stochastic background using data from Advanced LIGO's second observing run, Phys. Rev. D 100, 061101 (2019).

[8] L. Boco, A. Lapi, S. Goswami, F. Perrotta, C. Baccigalupi, and L. Danese, Merging rates of compact binaries in Galaxies: Perspectives for gravitational wave detections, Astrophys. J. 881, 157 (2019).

[9] X.-J. Zhu, E. J. Howell, D. G. Blair, and Z.-H. Zhu, On the gravitational wave background from compact binary coalescences in the band of ground-based interferometers, Mon. Not. R. Astron. Soc. 431, 882 (2013).

[10] R. Smith and E. Thrane, Optimal Search for an Astrophysical Gravitational-Wave Background, Phys. Rev. X 8, 021019 (2018).

[11] C. Talbot and E. Thrane, Measuring the binary black hole mass spectrum with an astrophysically motivated parameterization, Astrophys. J. 856, 173 (2018).

[12] J. J. Blanco-Pillado, K. D. Olum, and X. Siemens, New limits on cosmic strings from gravitational wave observation, Phys. Lett. B 778, 392 (2018).

[13] C. Ringeval and T. Suyama, Stochastic gravitational waves from cosmic string loops in scaling, J. Cosmol. Astropart. Phys. 12 (2017) 027.

[14] V. Cardoso and P. Pani, Testing the nature of dark compact objects: A status report, Living Rev. Relativity 22, 4 (2019).

[15] T. Regimbau and J. A. de Freitas Pacheco, Gravitational wave background from magnetars, Astron. Astrophys. 447, 1 (2006).

[16] B. P. Abbott et al., An upper limit on the stochastic gravitational-wave background of cosmological origin, Nature (London) 460, 990 (2009).

[17] M. C. Guzzetti, N. Bartolo, M. Liguori, and S. Matarrese, Gravitational waves from inflation, Riv. Nuovo Cimento 39, 399 (2016).

[18] M. Hindmarsh, S. J. Huber, K. Rummukainen, and D. J. Weir, Shape of the acoustic gravitational wave power 
spectrum from a first order phase transition, Phys. Rev. D 96, 103520 (2017); Erratum, 101, 089902 (2020).

[19] T. Callister, M. Fishbach, D. Holz, and W. Farr, Shouts and murmurs: Combining individual gravitational-wave sources with the stochastic background to measure the history of binary black hole mergers, Astrophys. J. Lett. 896, L32 (2020).

[20] P. M. Meyers, K. Martinovic, N. Christensen, and M. Sakellariadou, Detecting a stochastic gravitational-wave background in the presence of correlated magnetic noise, Phys. Rev. D 102, 102005 (2020).

[21] V. Mandic, E. Thrane, S. Giampanis, and T. Regimbau, Parameter Estimation in Searches for the Stochastic Gravitational-Wave Background, Phys. Rev. Lett. 109, 171102 (2012).

[22] T. Callister, A. S. Biscoveanu, N. Christensen, M. Isi, A. Matas, O. Minazzoli, T. Regimbau, M. Sakellariadou, J. Tasson, and E. Thrane, Polarization-Based Tests of Gravity with the Stochastic Gravitational-Wave Background, Phys. Rev. X 7, 041058 (2017).

[23] L. Tsukada, T. Callister, A. Matas, and P. Meyers, First search for a stochastic gravitational-wave background from ultralight bosons, Phys. Rev. D 99, 103015 (2019).

[24] J. Aasi et al., Advanced LIGO, Classical Quantum Gravity 32, 115012 (2015).

[25] F. Acernese et al., Advanced Virgo: A second-generation interferometric gravitational wave detector, Classical Quantum Gravity 32, 024001 (2015).

[26] M. Punturo et al., The einstein telescope: A third-generation gravitational wave observatory, Classical Quantum Gravity 27, 194002 (2010).

[27] B. P. Abbott et al., Exploring the sensitivity of next generation gravitational wave detectors, Classical Quantum Gravity 34, 044001 (2017).

[28] D. Reitze et al., The US program in ground-based gravitational wave science: Contribution from the LIGO Laboratory, Bull. Am. Astron. Soc. 51, 141 (2019).

[29] T. Regimbau, M. Evans, N. Christensen, E. Katsavounidis, B. Sathyaprakash, and S. Vitale, Digging Deeper: Observing Primordial Gravitational Waves Below the BinaryBlack-Hole-Produced Stochastic Background, Phys. Rev. Lett. 118, 151105 (2017).

[30] S. Sachdev, T. Regimbau, and B. S. Sathyaprakash, Subtracting compact binary foreground sources to reveal primordial gravitational-wave backgrounds, Phys. Rev. D 102, 024051 (2020).

[31] C. Cutler and J. Harms, Big bang observer and the neutronstar-binary subtraction problem, Phys. Rev. D 73, 042001 (2006).

[32] J. Harms, C. Mahrdt, M. Otto, and M. Prieß, Subtractionnoise projection in gravitational-wave detector networks, Phys. Rev. D 77, 123010 (2008).

[33] A. Sharma and J. Harms, Searching for cosmological gravitational-wave backgrounds with third-generation detectors in the presence of an astrophysical foreground, Phys. Rev. D 102, 063009 (2020).

[34] A. Vilenkin and E. P. S. Shellard, Cosmic Strings and Other Topological Defects (Cambridge University Press, Cambridge, England, 2000).
[35] E. Witten, Cosmic separation of phases, Phys. Rev. D 30, 272 (1984).

[36] C. J. Hogan, Gravitational radiation from cosmological phase transitions, Mon. Not. R. Astron. Soc. 218, 629 (1986).

[37] T. Callister, L. Sammut, S. Qiu, I. M. el, and E. Thrane, Limits of Astrophysics with Gravitational-Wave Backgrounds, Phys. Rev. X 6, 031018 (2016).

[38] A. Saffer and K. Yagi, Parameter estimation for tests of general relativity with the astrophysical stochastic gravitational wave background, Phys. Rev. D 102, 024001 (2020).

[39] R. Jeannerot, J. Rocher, and M. Sakellariadou, How generic is cosmic string formation in SUSY GUTs, Phys. Rev. D 68 , 103514 (2003).

[40] T. Damour and A. Vilenkin, Gravitational radiation from cosmic (super)strings: Bursts, stochastic background, and observational windows, Phys. Rev. D 71, 063510 (2005).

[41] L. Lentati et al., European pulsar timing array limits on an isotropic stochastic gravitational-wave background, Mon. Not. R. Astron. Soc. 453, 2577 (2015).

[42] X. Siemens, V. Mandic, and J. Creighton, Gravitational Wave Stochastic Background from Cosmic (Super)Strings, Phys. Rev. Lett. 98, 111101 (2007).

[43] Y. Gouttenoire, G. Servant, and P. Simakachorn, Beyond the standard models with cosmic strings, J. Cosmol. Astropart. Phys. 07 (2020) 032.

[44] J. J. Blanco-Pillado, K. D. Olum, and B. Shlaer, The number of cosmic string loops, Phys. Rev. D 89, 023512 (2014).

[45] L. Lorenz, C. Ringeval, and M. Sakellariadou, Cosmic string loop distribution on all length scales and at any redshift, J. Cosmol. Astropart. Phys. 10 (2010) 003.

[46] B. Von Harling, A. Pomarol, O. Pujolàs, and F. Rompineve, Peccei-Quinn phase transition at LIGO, J. High Energy Phys. 04 (2020) 195.

[47] C. Caprini, M. Hindmarsh, S. Huber, T. Konstandin, J. Kozaczuk, G. Nardini, J. Miguel No, A. Petiteau, P. Schwaller, G. Servant, and D. J. Weir, Science with the space-based interferometer eLISA. II: Gravitational waves from cosmological phase transitions, J. Cosmol. Astropart. Phys. 04 (2016) 001.

[48] S. J. Huber and T. Konstandin, Gravitational wave production by collisions: More bubbles, J. Cosmol. Astropart. Phys. 09 (2008) 022.

[49] M. Hindmarsh, S. J. Huber, K. Rummukainen, and D. J. Weir, Gravitational Waves from the Sound of a First Order Phase Transition, Phys. Rev. Lett. 112, 041301 (2014).

[50] M. Hindmarsh, S. . Huber, K. Rummukainen, and D. J. Weir, Numerical simulations of acoustically generated gravitational waves at a first order phase transition, Phys. Rev. D 92, 123009 (2015).

[51] C. Caprini, R. Durrer, and G. Servant, Gravitational wave generation from bubble collisions in first-order phase transitions: An analytic approach, Phys. Rev. D 77, 124015 (2008).

[52] R. Jinno and M. Takimoto, Gravitational waves from bubble collisions: An analytic derivation, Phys. Rev. D 95, 024009 (2017).

[53] D. . Weir, Gravitational waves from a first order electroweak phase transition: A brief review, Phil. Trans. R. Soc. A 376, 20170126 (2018). 
[54] J.. Romano and N. J. Cornish, Detection methods for stochastic gravitational-wave backgrounds: A unified treatment, Living Rev. Relativity 20, 2 (2017).

[55] A. Parida, S. Mitra, and S. Jhingan, Component separation of a isotropic gravitational wave background, J. Cosmol. Astropart. Phys. 04 (2016) 024.

[56] S. Kuroyanagi, T. Chiba, and T. Takahashi, Probing the Universe through the stochastic gravitational wave background, J. Cosmol. Astropart. Phys. 11 (2018) 038.

[57] B. P. Abbott et al., Prospects for observing and localizing gravitational-wave transients with Advanced LIGO, Advanced Virgo and KAGRA, Living Rev. Relativity 21, 3 (2018).

[58] S. Hild et al., Sensitivity studies for third-generation gravitational wave observatories, Classical Quantum Gravity 28, 094013 (2011).

[59] B. P. Abbott et al., Exploring the sensitivity of next generation gravitational wave detectors, Classical Quantum Gravity 34, 044001 (2017).

[60] S. Biscoveanu, C. Talbot, E. Thrane, and R. Smith, Measuring the Primordial Gravitational-Wave Background in the Presence of Astrophysical Foregrounds, Phys. Rev. Lett. 125, 241101 (2020).

[61] S. Mukherjee and J. Silk, Time-dependence of the astrophysical stochastic gravitational wave background, Mon. Not. R. Astron. Soc. 491, 4690 (2020).

[62] D. Meacher, E. Thrane, and T. Regimbau, Statistical properties of astrophysical gravitational-wave backgrounds, Phys. Rev. D 89, 084063 (2014).

[63] D. Meacher, K. Cannon, C. Hanna, T. Regimbau, and B. S. Sathyaprakash, Second Einstein telescope mock data and science challenge: Low frequency binary neutron star data analysis, Phys. Rev. D 93, 024018 (2016).

[64] P. Auclair et al., Probing the gravitational wave background from cosmic strings with LISA, J. Cosmol. Astropart. Phys. 04 (2020) 034.

[65] P. Amaro-Seoane et al., Laser interferometer space antenna, arXiv:1702.00786.
[66] C. Caprini, D. G. Figueroa, R. Flauger, G. Nardini, M. Peloso, M. Pieroni, A. Ricciardone, and G. Tasinato, Reconstructing the spectral shape of a stochastic gravitational wave background with LISA, J. Cosmol. Astropart. Phys. 11 (2019) 017.

[67] M. R. Adams and N. J. Cornish, Detecting a stochastic gravitational wave background in the presence of a galactic foreground and instrument noise, Phys. Rev. D 89, 022001 (2014).

[68] M. R. Adams and N. J. Cornish, Discriminating between a stochastic gravitational wave background and instrument noise, Phys. Rev. D 82, 022002 (2010).

[69] M. Tinto and S. V. Dhurandhar, Time-delay interferometry, Living Rev. Relativity 17, 6 (2014).

[70] M. Gasperini and M. Giovannini, Dilaton contributions to the cosmic gravitational wave background, Phys. Rev. D 47, 1519 (1993).

[71] E. Thrane, N. Christensen, and R. Schofield, Correlated magnetic noise in global networks of gravitational-wave interferometers: Observations and implications, Phys. Rev. D 87, 123009 (2013).

[72] F. Amann et al., Site-selection criteria for the Einstein telescope, Rev. Sci. Instrum. 91, 094504 (2020).

[73] J. D. Hunter, Matplotlib: A 2d graphics environment, Comput. Sci. Eng. 9, 90 (2007).

[74] S. van der Walt, S. C. Colbert, and G. Varoquaux, The NumPy array: A structure for efficient numerical computation, Comput. Sci. Eng. 13, 22 (2011).

[75] P. Virtanen et al. (scipy 1.0 Contributors), scipy 1.0: Fundamental algorithms for scientific computing in PYTHON, Nat. Methods 17, 261 (2020).

[76] G. Ashton et al., BILBY: A user-friendly Bayesian inference library for gravitational-wave astronomy, Astrophys. J. Suppl. 241, 27 (2019).

[77] J. Veitch, W. Del Pozzo, M. Cody, M. Pitkin, and ed1d1a8d, johnveitch/cpnest: Minor optimisation (2017).

[78] S. R. Hinton, ChainConsumer, J. Open Source Software 1, 00045 (2016).

[79] M. Waskom et al., seaborn: v0.5.0 (2014). 